\title{
Universal X-ray emissivity of the stellar population in early-type galaxies: unresolved X-ray sources in NGC 3379
}

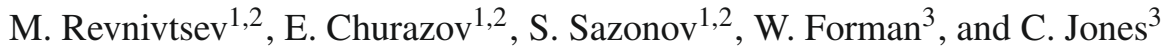 \\ ${ }^{1}$ Max-Planck-Institute für Astrophysik, Karl-Schwarzschild-Str. 1, 85740 Garching bei München, Germany \\ e-mail: mikej@mpa-garching.mpg.de \\ 2 Space Research Institute, Russian Academy of Sciences, Profsoyuznaya 84/32, 117997 Moscow, Russia \\ ${ }^{3}$ Harvard-Smithsonian Center for Astrophysics, 60 Garden Street, Cambridge, MA 02138, USA
}

Received 2 April 2008 / Accepted 25 June 2008

\begin{abstract}
We use deep Chandra observations to measure the emissivity of the unresolved X-ray emission in the elliptical galaxy NGC 3379. After elimination of bright, low-mass X-ray binaries with luminosities $\gtrsim 10^{36} \mathrm{erg} \mathrm{s}^{-1}$, we find that the remaining unresolved X-ray emission is characterized by an emissivity per unit stellar mass $L_{\mathrm{x}} / M_{*} \sim 8.2 \times 10^{27} \mathrm{erg} \mathrm{s}^{-1} M_{\odot}^{-1}$ in the $0.5-2 \mathrm{keV}$ energy band. This value is in good agreement with those previosuly determined for the dwarf elliptical galaxy M 32, the bulge of the spiral galaxy M 31 and the Milky Way, as well as with the integrated X-ray emissivity of cataclysmic variables and coronally active binaries in the Solar neighborhood. This strongly suggests that i) the bulk of the unresolved X-ray emission in NGC 3379 is produced by its old stellar population; and ii) the old stellar populations in all galaxies can be characterized by a universal value of X-ray emissivity per unit stellar mass or per unit $K$ band luminosity.
\end{abstract}

Key words. galaxies: bulges - X-rays: binaries - X-rays: galaxies - X-rays: ISM - galaxies: fundamental parameters

\section{Introduction}

Non-active galaxies produce X-ray emission that generally consists of a diffuse component originating in hot interstellar gas and emission from point-like sources (e.g. Trinchieri \& Fabbiano 1985). The diffuse X-ray emission is a powerful tool that provides a record of past star formation and central massive black hole activity through their effects on the interstellar medium (e.g. Heckman et al. 1990; Loewenstein \& Mathews 1991; Strickland et al. 2000; Churazov et al. 2001; Bîrzan et al. 2004), and to measure total (including dark matter) masses of elliptical galaxies (Forman et al. 1985).

Luminous $\left(L_{\mathrm{x}} \gtrsim 10^{35}-10^{36} \mathrm{erg} \mathrm{s}^{-1}\right)$ compact sources, including low- and high-mass X-ray binaries (LMXBs and HMXBs, respectively) can be individually detected in nearby galaxies with Chandra and XMM-Newton. Since the measured luminosity functions of both LMXBs and HMXBs are significantly flatter than $\mathrm{d} N / \mathrm{d} L_{\mathrm{x}} \propto L_{\mathrm{x}}^{-2}$ at low luminosities (Gilfanov 2004), many studies assumed that any remaining unresolved $\mathrm{X}$-ray emission in galaxies must originate in hot interstellar gas (e.g. Shirey et al. 2001; Strickland et al. 2004, to name but a few).

However, it was recently recognized that weak X-ray sources with luminosities $10^{28}-10^{34} \mathrm{erg} \mathrm{s}^{-1}$ located in the Solar vicinity, in particular cataclysmic variables $(\mathrm{CVs})$ and coronally active binaries (ABs), together produce a considerable X-ray luminosity per unit stellar mass (Sazonov et al. 2006), which allows one to explain the apparently diffuse X-ray emission distributed over our Galaxy (the so-called Galactic Ridge X-ray Emission) as the superposition of a great number of such low-luminosity sources (Revnivtsev et al. 2006). Since CVs and ABs (and LMXBs) are not associated with ongoing or recent star formation, it is reasonable to expect that such objects should produce, in all galaxies, approximately the same amount of X-ray emission per unit stellar mass as in the Milky Way.

A particularly important contribution from unresolved point sources may be expected in the soft X-ray band (0.5-2 keV). Indeed, the cumulative emissivity of low-luminosity sources in the Solar neighborhood is estimated at $\mathrm{d} L_{\mathrm{x}} / M_{*} \sim 1-3 \times$ $10^{28} \mathrm{erg} \mathrm{s}^{-1}$ (Sazonov et al. 2006), which for a galaxy of mass $M_{*}$ implies a total luminosity of a few $\times 10^{39}\left(M_{*} / 10^{11} M_{\odot}\right) \mathrm{erg} \mathrm{s}^{-1}$ in unresolved soft X-ray emission, which is just a few times smaller than the expected cumulative luminosity of bright LMXBs (Gilfanov 2004). In fact, the possibility that unresolved point sources might provide an important contribution to the X-ray luminosity of galaxies was discussed in several early works (Pellegrini \& Fabbiano 1994; Irwin \& Sarazin 1998) but was not supported by quantitative estimates (see however some estimates in Pellegrini et al. 2007). Importantly, both in coronally active stars and in cataclysmic variables (i.e. accreting white dwarfs), we are dealing with thermal X-ray emission from an optically thin plasma, with temperatures $10^{6}-10^{7} \mathrm{~K}$ and $10^{7}-10^{8} \mathrm{~K}$, respectively, similar to that produced by a hot, multi-temperature interstellar gas. In particular, X-ray spectra of coronal stars show a prominent peak at energies $0.6-0.8 \mathrm{keV}$ due to a blend of atomic lines, and the same is expected for interstellar gas with temperatures $\sim 0.5-1 \mathrm{keV}$. Therefore, separating the collective X-ray emission of faint point sources from truly diffuse emission produced by hot interstellar gas is hardly possible by means of X-ray spectroscopy alone.

One might hope, however, to be able to predict the contribution of unresolved point sources to the X-ray emission of a given 


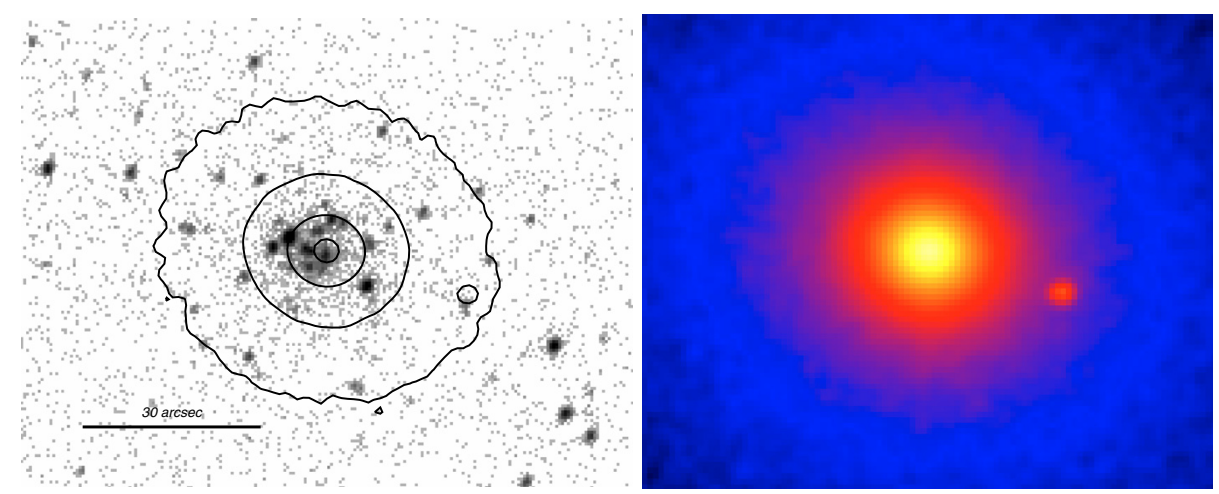

Fig. 1. Raw Chandra image (left panel) of NGC 3379 compared with the near-infrared ( $K$-band) image of the galaxy (right panel). Contours in the left image are logarithmically spaced near-infrared brightness isophotes.

galaxy using the measured cumulative luminosity (per unit stellar mass) of faint X-ray sources in the Solar vicinity. But how well does this locally determined value characterize other galaxies? Is the cumulative X-ray emissivity of faint sources strongly sensitive to the properties (age, metallicity, etc.) of the stellar population of a given galaxy? To answer these questions, we selected several nearby galaxies with available Chandra observations that are likely to be sufficiently gas poor for the bulk of their unresolved X-ray emission to be due to unresolved point sources. The first galaxy studied was the dwarf elliptical galaxy M 32, for which we demonstrated (Revnivtsev et al. 2007) that the soft X-ray emissivity (with bright LMXBs excluded) per unit stellar mass is compatible with the combined emissivity of CVs and ABs in the Solar vicinity (Sazonov et al. 2006).

In the present paper, we extend this study to a moderately massive elliptical galaxy, NGC 3379. We focus on studying the properties of the unresolved X-ray component in the soft band $0.5-2 \mathrm{keV}$, where the sensitivity of Chandra is maximal. We also discuss the broad-band spectral properties of this emission and the implications for early-type galaxies in general. The unresolved X-ray component of NGC 3379 was previously studied by David et al. (2005) and Fukazawa et al. (2006) using smaller datasets. Application of the usual assumption that the hot $\mathrm{X}$-ray emitting interstellar gas is in hydrostatic equilibrium in this galaxy implies an unusually high mass-to-light ratio, which led to the suggestion (e.g. Fukazawa et al. 2006) that a significant contribution to the observed X-ray flux of the galaxy comes from unresolved point sources. We argue in this paper that the bulk of this emission is likely the superposition of faint point sources, CVs and ABs. 2003).

We adopt a distance to NGC 3379 of $9.8 \mathrm{Mpc}$ (Jensen et al.

\section{Data analysis}

We used all publicly available Chandra observations of NGC 3379 (ObsIDs 1587, 4692, 7073, 7074, 7075, 7076), which in total provide approximately $340 \mathrm{ks}$ of useful exposure time.

The Chandra data were reduced following a standard procedure fully described in Vikhlinin et al. (2005). The detector background was modeled using the stowed dataset (http:// cxc.harvard.edu/contrib/maxim/stowed). To additionally check the accuracy of the background subtraction, we also measured the detector background in regions away from NGC 3379.
Point sources were detected in the $0.5-7 \mathrm{keV}$ energy band using the wavelet decomposition package wvdecomp of ZHTOOLS (Vikhlinin et al. 1998) ${ }^{1}$. To study the unresolved X-ray emission of NGC 3379, we masked regions with radii $2.46^{\prime \prime}$ around the detected point sources, which ensures removal of more than $98 \%$ of the flux from these sources in the region of our study $\left(<100^{\prime \prime}\right.$ around the aimpoint of Chandra).

In our the spectral analysis, we adopted a Galactic photoabsorption column density toward NGC $3379 n_{\mathrm{H}} L=2.7 \times$ $10^{20} \mathrm{~cm}^{-2}$ (Dickey \& Lockman 1990) and all fluxes quoted below are corrected for this absorption.

\section{Unresolved X-ray emission in NGC 3379}

A conservative source detection threshold of 10 net counts corresponds for the analyzed (relatively long exposure) dataset to a flux $\sim 10^{-16} \mathrm{erg} \mathrm{s}^{-1} \mathrm{~cm}^{-2}$ in the energy band $0.5-7 \mathrm{keV}$. Given the adopted NGC 3379 distance, this allows us to detect and remove point sources with luminosities higher than $\sim 2 \times 10^{36} \mathrm{erg} \mathrm{s}^{-1}$.

\subsection{Properties of the unresolved $X$-ray emission}

In Fig. 1 we compare the raw Chandra image of NGC 3379, and the near-infrared ( $K$-band) image of the galaxy obtained from the 2MASS Large Galaxy Atlas (Jarrett et al. 2003, see also http://irsa.ipac.caltech.edu/applications/ 2MASS/LGA/). In the following analysis, we assumed that the total brightness of the galaxy is $K=6.27$ (taken from the 2MASS Large Galaxy Atlas). For a distance modulus $m-M=29.96$ (Jensen et al. 2003), the total near-infrared luminosity is $L_{K}=$ $6.8 \times 10^{10} L_{\odot}$. Using the color dependent mass-to-light ratio $M / L_{K}=0.84$ determined for $B-V=0.96$ from Bell et al. (2003), we further estimated the stellar mass of NGC 3379 at $M_{*} \approx 5.7 \times 10^{10} M_{\odot}$.

In Fig. 2 we compare the radial surface brightness profiles of the unresolved X-ray emission (i.e., excuding detected point sources) with the near-infrared light of NGC 3379. In Fig. 3, we show the energy spectrum of the unresolved X-ray emission collected by Chandra within the central region of NGC 3379 of radius $33^{\prime \prime}$, which is approximately the effective radius of the galaxy in the near-infrared spectral band.

One can see from Fig. 2 that the unresolved X-ray $(0.5-2 \mathrm{keV})$ emission very closely follows the near-infrared

\footnotetext{
${ }^{1}$ http://hea-www . harvard. edu/saord/zhtools/
} 


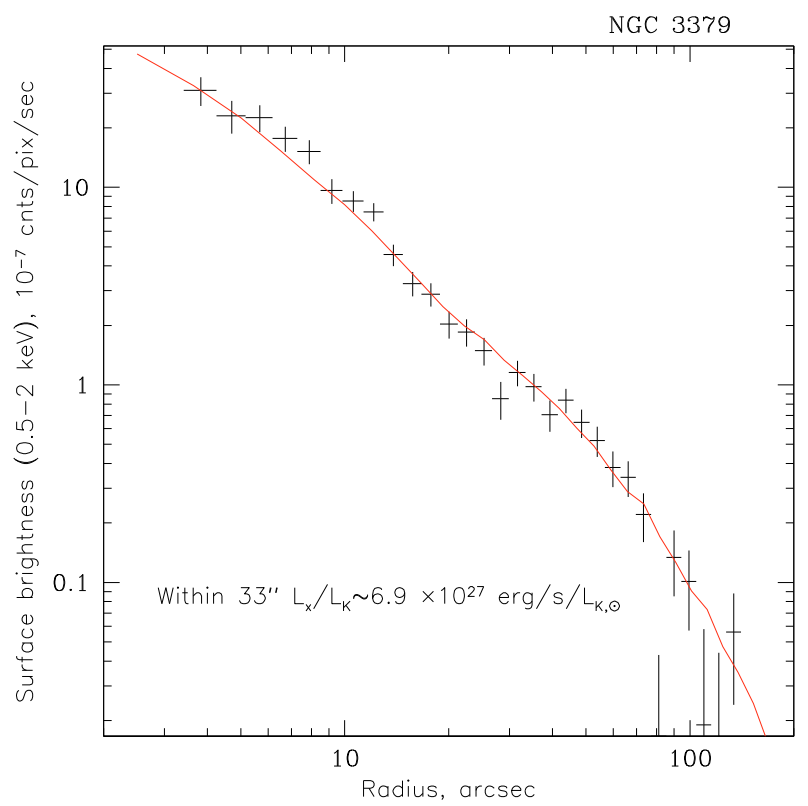

Fig. 2. Radial surface brightness profile of NGC 3379 in the $0.5-2 \mathrm{keV}$ energy band. The contribution of point sources with luminosities $>2 \times$ $10^{36} \mathrm{erg} \mathrm{s}^{-1}$ has been subtracted. The solid line shows the radial profile of near-infrared ( $K$-band) surface brightness, rescaled by the factor $L_{0.5-2 \mathrm{keV}} / L_{K}=6.9 \times 10^{27} \mathrm{erg} \mathrm{s}^{-1} L_{K, \odot}^{-1}$. The agreement between the $\mathrm{X}$-ray and $K$-band profiles is excellent and supports the stellar origin of the soft X-ray emission.

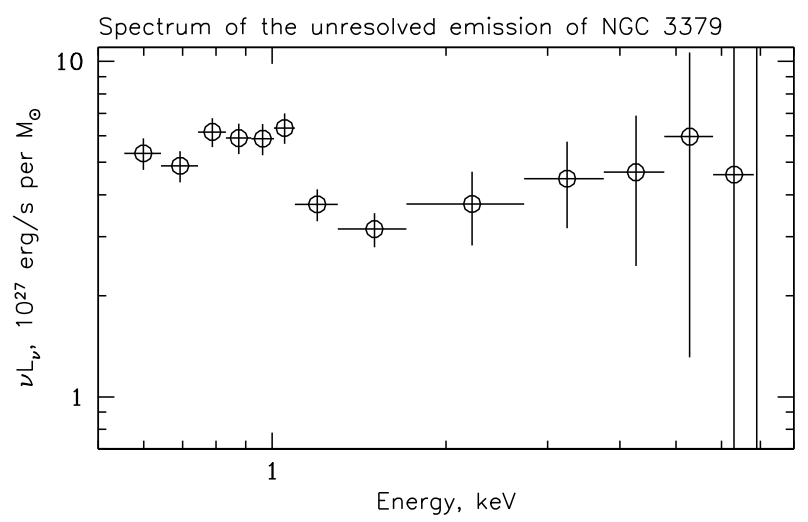

Fig. 3. Spectrum of the unresolved X-ray emission of NGC 3379, normalized to the stellar mass contained in the $33^{\prime \prime}$ central region.

light in NGC 3379, which implies that this emission is characterized by a constant ratio of X-ray and $K$-band emissivities, or by constant X-ray emissivity per unit stellar mass. In the region $<33^{\prime \prime}$ around the center of the galaxy:

$$
\begin{aligned}
& \frac{L_{0.5-2 \mathrm{keV}}}{L_{K}}=(6.9 \pm 0.7) \times 10^{27} \mathrm{erg}^{-1} \mathrm{~s}^{-1} L_{K, \odot}^{-1}, \\
& \frac{L_{0.5-2 \mathrm{keV}}}{M_{*}}=8.2 \pm 0.8( \pm 2.5) \times 10^{27} \mathrm{erg}^{-1} \mathrm{~s}^{-1} M_{\odot}^{-1} .
\end{aligned}
$$

The quoted uncertanties in Eqs. (1) and (2) are derived from the statistical errors in the measured X-ray flux. In addition, we assumed a $30 \%$ uncertainty in the $L_{K}$ to $M_{*}$ conversion (see e.g. Bell et al. 2003); the corresponding systematic uncertainty is given in parentheses in Eq. (2).

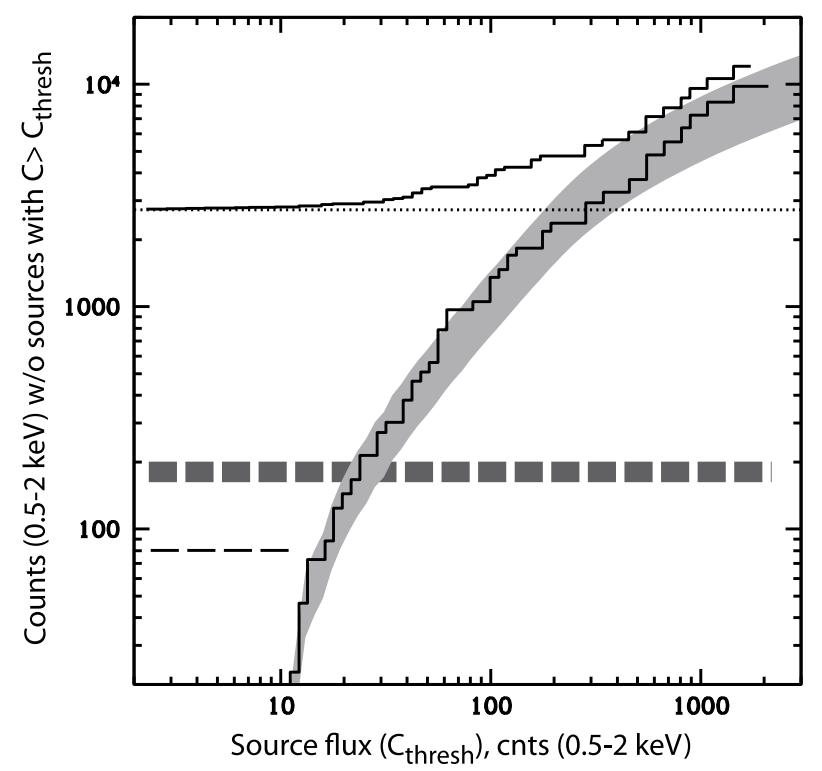

Fig. 4. Number of counts detected by Chandra in the $0.5-2 \mathrm{keV}$ energy band within the central 33" radius region of NGC 3379 as a function of the threshold above which point sources were masked (upper solid histogram). The lower histogram shows $N_{\text {thresh }}$, the cumulative number of counts from detected point sources (i.e. those brighter than 10 counts) that are weaker than the given threshold. The gray area shows the predicted value of $N_{\text {thresh }}$ assuming that all the detected sources are LMXBs and is based on the luminosity function of LMXBs in nearby galaxies integrated above $L_{\mathrm{x}}=2 \times 10^{36} \mathrm{erg} \mathrm{s}^{-1}$; the thickness of the gray band corresponds to the variance in the normalization of the LMXB luminosity function between different galaxies (Gilfanov 2004). Note that an LMXB with a typical power-law spectrum with photon index $\Gamma=1.6$ (Irwin et al. 2003) providing 10 net Chandra counts in the energy band $0.5-2 \mathrm{keV}$ will have an X-ray luminosity $\sim 2 \times 10^{36} \mathrm{erg} \mathrm{s}^{-1}$ in the energy band $0.5-7 \mathrm{keV}$. The thin dashed line shows the expected flux of LMXBs with luminosities $10^{35}-2 \times 10^{36} \mathrm{erg} \mathrm{s}^{-1}$. The thick gray horizontal dashed line denotes the residual flux outside the $2.46^{\prime \prime}$ radius circles used to mask bright sources.

\subsection{Remaining contribution of $L M X B$ s below the detection threshold?}

Since NGC 3379 is an elliptical galaxy with virtually no ongoing star formation, essentially all of the detected bright X-ray sources (with luminosities above $\sim 2 \times 10^{36} \mathrm{erg} \mathrm{s}^{-1}$ ) are expected to be low mass X-ray binaries. Could the remaining unresolved emission be due to LMXBs with luminosities below our detection threshold? To investigate this possibility, we allowed the threshold for masking point sources to vary and calculated the total number of counts in the masked $0.5-2 \mathrm{keV}$ Chandra image as a function of the threshold (Fig. 4). One can see that this dependence flattens at luminosities below $\sim 2 \times 10^{37} \mathrm{erg} \mathrm{s}^{-1}$, confirming the presence of unresolved X-ray emission.

If we assume that the average luminosity function of LMXBs in nearby galaxies (Gilfanov 2004) continues below $10^{36} \mathrm{erg} \mathrm{s}^{-1}$ with the same slope as measured in the range $10^{36}-10^{37} \mathrm{erg} \mathrm{s}^{-1}$, we find (see Fig. 4 and also Fig. 6 in Revnivtsev et al. 2007) that unresolved LMXBs $\left(L_{0.5-7 \mathrm{keV}} \lesssim 2 \times 10^{36} \mathrm{erg} \mathrm{s}^{-1}\right)$, whose collective emissivity per unit stellar mass is estimated to be $\$ 10^{27} \mathrm{erg} \mathrm{s}^{-1} M_{\odot}^{-1}$, cannot account for more than $\sim 3 \%$ of the unresolved X-ray emission of NGC 3379. We also estimate that less than $\sim 8 \%$ of the unresolved emission can result from our incomplete removal of bright sources (see Fig. 4). 

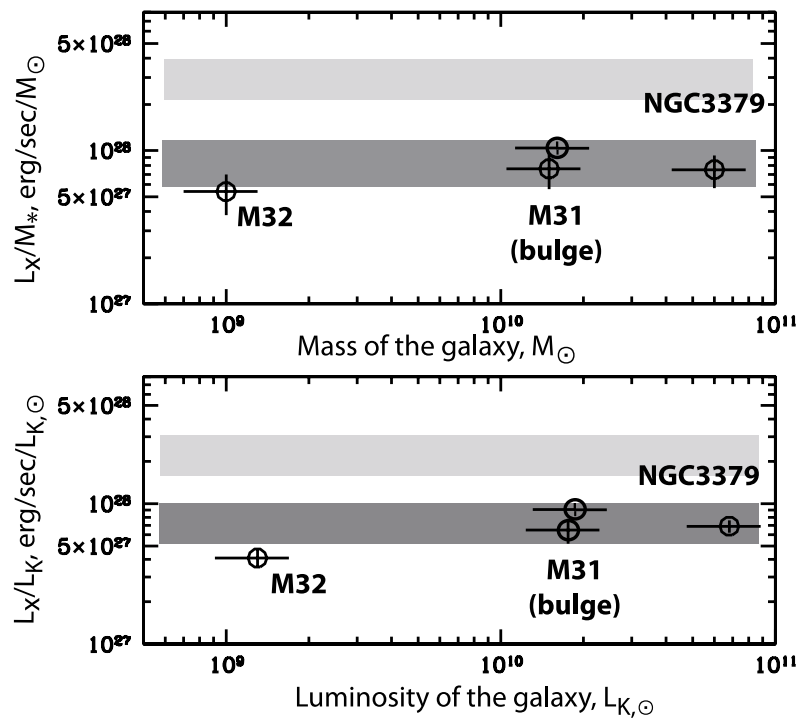

Fig. 5. Emissivities per unit stellar mass (upper panel) and per unit $K$-band luminosity (lower panel) of the unresolved soft X-ray $(0.5-2 \mathrm{keV})$ emission in three galaxies: M 32, the bulge of M 31 and NGC 3379 (see text).

\subsection{Nature of the unresolved emission - superposition of weak sources}

What is then the origin of the unresolved soft X-ray $(0.5-2 \mathrm{keV})$ emission of NGC 3379? Previous studies assumed that it is produced by hot interstellar gas (e.g. David et al. 2005; Fukazawa et al. 2006; Pellegrini \& Ciotti 2006) and did not consider the possibility that weak $\left(L_{\mathrm{x}} \lesssim 10^{35} \mathrm{erg} \mathrm{s}^{-1}\right)$ undetectable X-ray sources might provide a significiant or dominant contribution.

We recently demonstrated (Revnivtsev et al. 2007) that the unresolved X-ray emission of the nearby dwarf elliptical galaxy M 32 has the same morphology as its near-infrared light and the X-ray emissivity per unit stellar mass proved to be comparable to the cumulative emissivity of faint X-ray sources - cataclysmic variables and coronally active binaries - in the solar neighborhood (Sazonov et al. 2006). Similarly, the unresolved $\mathrm{X}$-ray emission in the ridge of our Galaxy (Revnivtsev et al. 2006) and in the bulge of M 31 (Li \& Wang 2007) have comparable X-ray emissivities. The similarity of these unresolved X-ray emissivities (per solar mass) strongly suggests that their unresolved X-ray emission arises from numerous faint X-ray sources, mostly CVs and ABs, and that this emission is characterized by a nearly constant emissivity per unit stellar mass, similarly to the case of brighter X-ray sources - LMXBs.

The qualitatively and quantitatively similar results presented here for NGC 3379 lead us to conclude that also in this galaxy the bulk of the unresolved X-ray emission is produced by weak $\mathrm{X}$-ray sources, mostly $\mathrm{CVs}$ and $\mathrm{ABs}$, rather than by hot interstellar gas.

Figure 5 demonstrates that the emissivities of the unresolved soft X-ray emission in the elliptical galaxies NGC 3379 and M 32 and in the bulge of the spiral galaxy M 31 agree within a factor of 2 with each other and are compatible with the cumulative emissivity of CVs and $\mathrm{ABs}$ in the Solar neighborhood. For this figure the values for M 32 and NGC 3379 are adopted from Revnivtsev et al. (2007) and the current paper, respectively. The $L_{\mathrm{x}} / M_{*}$ values for the M 31 bulge are adopted from Li \& Wang (2007) and Bogdan \& Gilfanov (2008) and their conversion to $L_{\mathrm{x}} / L_{K}$ assumed the mass-to-light ratio $M_{*} / L_{K}=0.85$. The darkgray stripe indicates the value and uncertainty of the cumulative
X-ray emissivity of cataclysmic variables and coronally active binary stars (both representing the old stellar population) in the Solar vicinity (Sazonov et al. 2006); the estimate shown by the light-gray area also includes the contribution of nearby young $(\ll 1$ Gyr) coronal stars (Sazonov et al. 2006), which are expected to be rare in early-type galaxies and galactic bulges. The conversion from $L_{\mathrm{x}} / M_{*}$ to $L_{\mathrm{x}} / L_{K}$ for the Solar vicinity values was done assuming $M_{*} / L_{K}=0.85$.

The spectral shape of the unresolved X-ray emission of NGC 3379 is broadly compatible with the combination of spectra of CVs and ABs in proportion, adopted from the Solar vicinity analysis by Sazonov et al. (2006). However, our knowledge of the relative contributions of $\mathrm{CVs}$ and $\mathrm{ABs}$ to the galactic emission in the $0.5-2.0 \mathrm{keV}$ energy band remains limited with considerable uncertainties (see e.g. Sazonov et al. 2006).

We note here that in the Solar vicinity an additional significant soft X-ray emission is produced by young coronal stars associated with recent star formation and the local estimate, including this contribution, is a factor of 2-3 larger than the emissivities found for M 32, NGC 3379 and for the M 31 bulge (although there is a large uncertainty in the local value, Sazonov et al. 2006). The lower emissivity of the three early-type galaxies/bulges is expected due to their predominantly old stellar populations, with a much lower abundance of young stars than in the Solar neighborhood.

\section{Universality of the X-ray emissivity of the old stellar population}

We argued above that the X-ray emissivities of the weak X-ray sources associated with the (old) stellar populations of the three nearby early-type galaxies/bulges (NGC 3379, M 31, M 32) are mutually compatible. However, it is natural to expect some variations in this emissivity. First, since the main mechanism of X-ray production in coronally active stars and cataclysmic variables is optically thin plasma emission, metal lines are expected to provide a considerable contribution in the energy band $0.5-2 \mathrm{keV}$ (e.g. the blend of lines at $\sim 0.8 \mathrm{keV}$ ). Hence the X-ray emissivity of a stellar population should depend on its metallicity.

Second, the age of a stellar population can affect its X-ray emissivity. For example, the X-ray luminosity of chromospherically active stars is known to be determined by the star's rotational velocity (see e.g Walter \& Bowyer 1981), which in turn may depend on stellar age. Single stars gradually slow down as they age, which leads to a monotonic decrease of their X-ray luminosity. The evolution of chromospherically active stars in binaries is significantly more complicated (see e.g. Eker et al. 2006), making it difficult to predict the evolution of their X-ray luminosity. Also, the orbital periods of accreting white dwarf binaries (which dominate the unresolved X-ray emission of galaxies at energies $>3-5 \mathrm{keV}$ ) decrease with time (until they reach the so-called period minimum, see e.g. Paczynski \& Sienkiewicz 1981). This is accompanied by a decreasing mass accretion rate (e.g. Rappaport et al. 1982). Therefore, one might anticipate the $\mathrm{X}$-ray luminosity of CVs to decrease with age.

Despite these possible effects, the $L_{\mathrm{x}} / M_{*}$ ratios found for the unresolved X-ray emission in M 32, NGC 3379 and in the bulge of M 31 agree with each other within a factor of 2. This suggests that the X-ray emissivity (per unit stellar mass or per unit near-infrared luminosity) averaged over these early-type galaxies/bulges may be regarded as a nearly universal property characterizing the old $(\gtrsim 1 \mathrm{Gyr})$ stellar population in all galaxies: $L_{0.5-2 \mathrm{keV}} / M_{*}=(7.0 \pm 2.9) \times 10^{27} \mathrm{erg} \mathrm{s}^{-1} M_{\odot}^{-1}$ and 

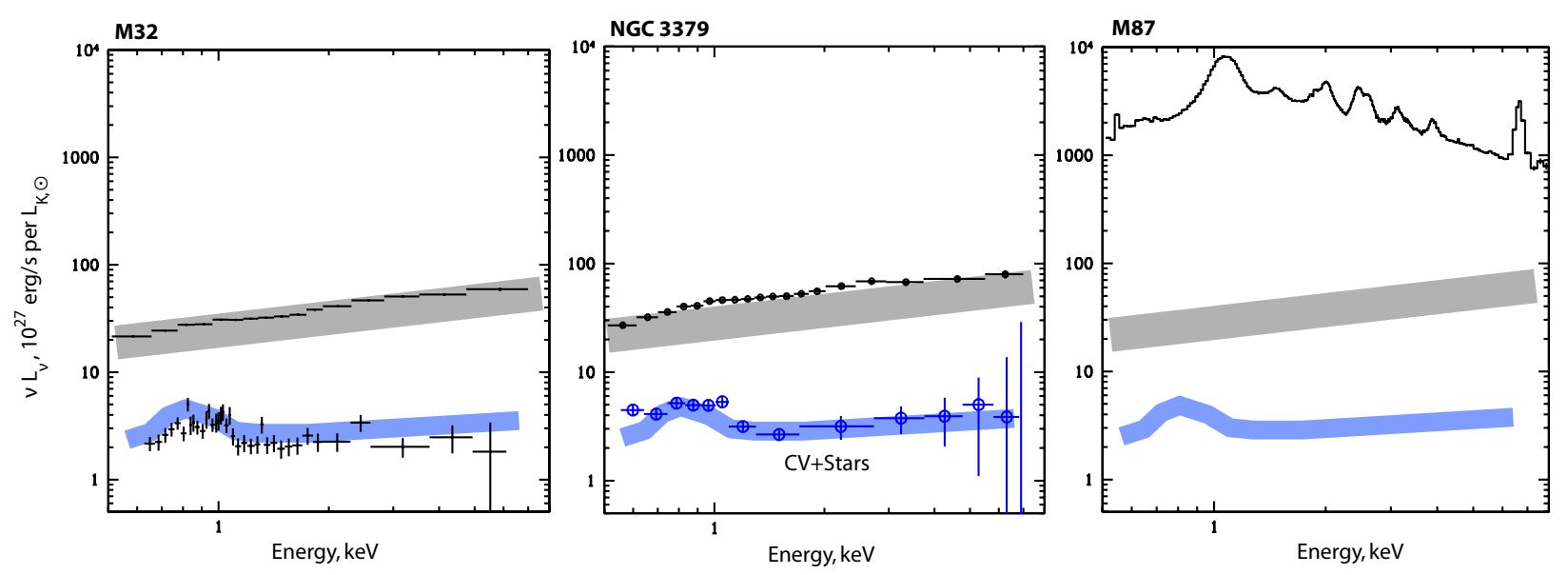

Fig. 6. Spectra of three elliptical galaxies of different masses normalized to their near-infrared ( $K$-band) luminosities. The upper sets of $C h a n d r a$ data points represent the total X-ray emission, collected within the effective radii of the galaxies ( 35" for M 32, 33" for NGC 3379 and $\sim 100^{\prime \prime}$ for M 87). The lower set of data points for M 32 and NGC 3379 represent their unresolved X-ray emission. The upper gray stripe indicates the expected contribution of LMXBs, which is a power law with photon index $\Gamma=1.6$ and normalization corresponding to the total $0.5-7 \mathrm{keV}$ emissivity of LMXBs with luminosities $L_{\mathrm{x}}>10^{36} \mathrm{erg} \mathrm{s}^{-1}$ calculated using the analytical approximation of the LMXB luminosity function from Gilfanov (2004). The thickness of this stripe indicates the scatter between the normalizations of the luminosity functions of different galaxies (Gilfanov 2004). The lower thick stripe shows a rough spectral model of the unresolved X-ray emission produced by weak point sources (see text), whose normalization is chosen to be the average over the three galaxies presented in Fig. 5. The thickness of the curve represents the scatter between the emissivities of the unresolved X-ray emission of those galaxies.

$L_{0.5-2 \mathrm{keV}} / L_{K}=(5.9 \pm 2.5) \times 10^{27} \mathrm{erg} \mathrm{s}^{-1} L_{K, \odot}^{-1}$. These values are the mean and the rms scatter of the values of $\mathrm{X}$-ray emissivity, measured in these three galaxies. It is important to note however that in regions of active star formation the cumulative X-ray emissivity of weak stellar-type sources may be significantly different from these values.

Since also the brighter X-ray sources belonging to the old stellar population - LMXBs - are characterized by similar universality (e.g. Gilfanov 2004), we might expect the X-ray emission of any early-type galaxy to include a resolvable (in nearby galaxies) component due to LMXBs and an unresolved component due to weak point sources, with the relative contributions of these components not varying significantly from one galaxy to another. In addition, there may be truly diffuse emission produced by hot interstellar gas.

To illustrate this point, we compare in Fig. 6 the X-ray $(0.5-7 \mathrm{keV})$ spectra of three elliptical galaxies with very different stellar masses and gas content. Specifically, we compare the total Chandra spectra of M $32\left(M_{*} \sim 10^{9} M_{\odot}\right)$, NGC 3379 $\left(M_{*} \sim 6 \times 10^{10} M_{\odot}\right)$ and the giant galaxy M $87\left(M_{*} \sim 4 \times\right.$ $\left.10^{11} M_{\odot}\right)$. For M 32 and NGC 3379 we also show the spectra of their unresolved X-ray emission.

The total emission of M 32 and NGC 3379 is clearly dominated by LMXBs and can be approximately described by a power-law spectrum with photon index $\Gamma=1.6$, a value typical of LMXB spectra in the $0.5-7 \mathrm{keV}$ energy band (Irwin et al. 2003). The unresolved $X$-ray emission of these galaxies, which we believe is the superposition of weak point sources, CVs and $\mathrm{ABs}$, has a spectrum that can be roughly approximated (using the Chandra spectrum of NGC 3379) by a combination of thermal plasma emission (mekal model in XSPEC) with temperature $k T=0.5 \mathrm{keV}$ and a Solar (Anders \& Grevesse 1989) abundance of heavy elements, and a power law with $\Gamma=1.9$. The ratio of the luminosities of the mekal and powerlaw components in the $0.5-2 \mathrm{keV}$ band was set to 2.03. These components add up so that their combined emissivities in the $0.5-2 \mathrm{keV}$ and $2-7 \mathrm{keV}$ bands are $L_{0.5-2 \mathrm{keV}} / M_{*}=(8.2 \pm 2.5) \times 10^{27} \mathrm{erg} \mathrm{s}^{-1} M_{\odot}^{-1}$ and $L_{2-7 \mathrm{keV}} / M_{*}=(6.3 \pm 2.5) \times 10^{27} \mathrm{erg} \mathrm{s}^{-1} M_{\odot}^{-1}$, respectively.
In the case of the giant galaxy M 87 in the Virgo cluster, most of the X-ray emission comes from the hot gas, while LMXBs and weak X-ray sources are expected to provide $\sim 1 \%$ and $\sim 0.1 \%$ of the total emission in $0.5-2 \mathrm{keV}$ energy band (see Fig. 6).

\section{Broad energy band picture for early-type galaxies: $0.5-100 \mathrm{keV}$}

The above discussion was restricted to the Chandra working energy band $(0.5-7 \mathrm{keV})$, with particular focus on the soft band (0.5-2 keV) where Chandra is most efficient. We now wish to extend this discussion to the study of early-type galaxies at photon energies up to $\sim 100 \mathrm{keV}$.

As was already discussed in the preceeding section, the $\mathrm{X}$-ray emission of an early-type galaxy is expected to consist of three main components: (i) emission from LMXBs, which may be at least partly resolved (depending on the instrument, exposure and distance to the galaxy); (ii) unresolved diffuse-like emission produced by cataclysmic variables and coronally active stars; and (iii) diffuse thermal emission of hot interstellar gas. There may also be additional contributions from e.g. nonthermal emission associated with cosmic rays or from X-ray sources representing young stellar populations (in particular HMXBs).

Below we discuss how the relative contributions of the main $\mathrm{X}$-ray emission components of an elliptical galaxy vary across the broad energy band $0.5-100 \mathrm{keV}$ and for illustration consider the X-ray spectrum of the giant gas rich elliptical galaxy M 87 (Fig. 7).

\section{1. $L M X B S$}

Low-mass X-ray binaries typically contribute at the level of $L_{\mathrm{x}} / M_{*} \sim 8 \times 10^{28} \mathrm{erg} \mathrm{s}^{-1} M_{\odot}^{-1}$ in $0.5-7 \mathrm{keV}$ energy band (e.g. Gilfanov 2004) and have very hard spectra at energies $<10 \mathrm{keV}$. At energies above $10 \mathrm{keV}$, the spectra of the brightest LMXBs $\left(L_{\mathrm{x}} \gtrsim 10^{37} \mathrm{erg} \mathrm{s}^{-1}\right)$, which contribute the most to the cumulative emissivity of LMXBs at $E<10 \mathrm{keV}$, have an exponential cutoff 


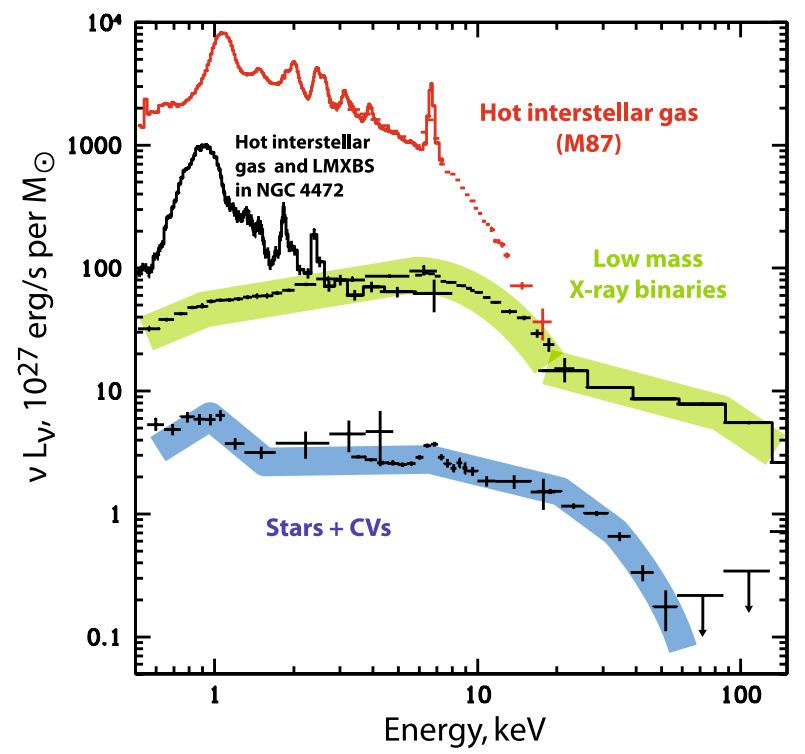

Fig. 7. Illustration of the composition of the X-ray spectrum of an elliptical galaxy from three main components (from the top to the bottom) hot interstellar gas (spectra of M 87 and NGC 4472); cumulative spectrum of emission of low mass X-ray binaries (spectrum of NGC 3379 and Milky Way, see text for details); cumulative spectrum of active stars and CVs (spectra of NGC 3379 and the Milky Way, see text for details).

(e.g. Mitsuda et al. 1984; Pavlinsky et al. 1994). Therefore, at still higher energies $(>20 \mathrm{keV})$ the less luminous LMXBs in the so-called low/hard spectral state dominate.

We show in Fig. 7 an expected broad-band spectrum of an entire galactic population of LMXBs. It is constructed of the Chandra spectrum of NGC 3379 at $E<7 \mathrm{keV}$ and the cumulative spectrum of the Galactic Center region (with the total mass of the enclosed stellar population $M_{*} \sim 10^{10} M_{\odot}$ ) obtained by Krivonos et al. (2007) with RXTE/PCA and INTEGRAL/IBIS at energies between 5 and $100 \mathrm{keV}$. Both parts of the spectrum were normalized by the corresponding stellar masses and the high-energy part was additionally multipled by a factor of 4 to match the data points at $E<7 \mathrm{keV}$. This additional rescaling can be justified partly because LMXBs are somewhat less abundant in the Milky Way than in nearby ellipticals (see e.g. Gilfanov 2004) and also because the observed number of sources which contribute the most to the cumulative flux in the Galactic Center region explored by Krivonos et al. (2007) is small. At energies 3-20 keV, it is LMXBs with $L_{\mathrm{x}} \sim 10^{37.5}-10^{38} \mathrm{erg} \mathrm{s}^{-1}$ (3 sources), and at energies $20-200 \mathrm{keV}$ it is LXMBs with $L_{\mathrm{x}} \sim 10^{36}-10^{37} \mathrm{erg} \mathrm{s}^{-1}$ (3-4 sources).

\section{2. $C V s$ and $A B s$}

In Fig. 7 we also show a composite spectrum of a galactic population of weaker point sources, including cataclysmic variables and coronally active binary stars. This spectrum is constucted from the Chandra spectrum of the unresolved emission of NGC 3379 at energies below $7 \mathrm{keV}$ and of the high-energy (3-100 keV) spectrum of the Galactic Ridge X-ray Emission measured by RXTE/PCA (Revnivtsev et al. 2006) and INTEGRAL/IBIS (Krivonos et al. 2007). This composite spectrum can be approximately described by a mekal+powerlaw model described in Sect. 4 with the multiplicative addition of a high-energy exponential cutoff, $\exp (-E / 18 \mathrm{keV})$.

\subsection{Hot interstellar gas}

More massive elliptical galaxies often reside in clusters or groups of galaxies and have large reservoirs of hot gas, which produces most of the X-ray emission of such galaxies. The best studied example is the giant elliptical galaxy M 87 in the central part of the Virgo cluster. In Fig. 7 we show the spectrum of M 87 collected by Chandra in the $0.5-7 \mathrm{keV}$ energy band within the optical effective radius of the galaxy $\left(\sim 100^{\prime \prime}\right)$, to which we attached the spectrum observed by RXTE/PCA in the $3-20 \mathrm{keV}$ band within the central $1^{\circ}$-radius region of $M 87$. To obtain a good match of the Chandra and RXTE data points, we multiplied the latter by a factor of 0.06 , which is approximately the ratio of the X-ray luminosities of the central $100^{\prime \prime}$ and $1^{\circ}$ regions of M 87 (e.g. Bohringer et al. 1994). Note that in the large region the emission is dominated by the thermal gas even more strongly than within the central $100^{\prime \prime}$ radius circle. Therefore, when the RXTE spectrum is renormalized to match the Chandra spectrum at energies of a few $\mathrm{keV}$, the contribution of the stellar population to the resulting spectrum shown in Fig. 7 is smaller than one would expect for the central $100^{\prime \prime}$ region.

From the above discussion and Fig. 7 we can draw the following conclusions:

- The 0.5-100 keV emission observed from a gas poor elliptical galaxy by a non-imaging (or poor angular resolution) instrument, such as $R X T E / P C A$, will be dominated by LMXBs, although weaker X-ray sources such as CVs and ABs will provide an important contribution (10-20\%) in the soft X-ray band (below $2 \mathrm{keV}$ ) and also near $20 \mathrm{keV}$.

- Future hard X-ray imaging instruments such as Nustar and Symbol-X should be able to resolve individual LMXBs in nearby galaxies. In this case, in gas poor elliptical galaxies there will be a residual diffuse-like hard X-ray component due to unresolved cataclysmic variables (coronally active stars provide a minor contribution at energies above $10 \mathrm{keV}$ ) resembling emission of diffuse thermal gas with a temperature of $\sim 20-30 \mathrm{keV}$.

- In gas rich galaxies like M 87, thermal emission of hot intergalactic gas strongly dominates at energies below $10 \mathrm{keV}$. At higher energies, however, the contribution of point sources can be significant or even dominant. In fact, as one can see from Fig. 7, LMXBs start to dominate in M 87 already at $\sim 20 \mathrm{keV}$, and if these LMXBs can be resolved by a future hard X-ray telescope then the remaining unresolved emission at energies 20-40 keV may have comparable contributions from hot interstellar gas and weak point sources (mostly CVs). Searches for nonthermal hard X-ray emission from brightest cluster galaxies, like M 87, must account for these other components.

\section{Summary and the future}

In this paper we have demonstrated that the X-ray emission of the gas poor elliptical galaxy NGC 3379 that remained unresolved after subtraction of bright X-ray sources (predominently LMXBs) can be explained as the cumulative emission of yet weaker descrete stellar-type sources, namely coronally active stars and cataclysmic variables. The correspondence of the measured X-ray emissivity (per unit stellar mass or per unit $K$-band luminosity) with the cumulative emissivity of such objects in the Solar vicinity indicates that there is no need for an additional contribution from hot interstellar gas in this galaxy.

The consistency (within a factor of 1.3) of the X-ray emissivities of faint discrete sources in the galaxies M 32, 
Milky Way, M 31 and NGC 3379, which differ in mass by more than a factor of 50, suggests that stellar-type sources (apart from bright LMXBs) in a given early-type galaxy of mass $M_{*}$ should produce a soft X-ray luminosity $L_{0.5-2 \mathrm{keV}} \sim 7 \times$ $10^{38}\left(M_{*} / 10^{11} M_{\odot}\right) \mathrm{erg} \mathrm{s}^{-1}$. Furthermore, since the majority of the contributing sources (CVs and ABs) are expected to be relatively old ( $\gtrsim 1 \mathrm{Gyr})$, they should provide a similar contribution also in late-type galaxies. However, in the latter case there might be an additional contribution from weak X-ray sources belonging to younger stellar populations, e.g. from rapidly rotating single coronal stars, as indicated by observations of the Solar vicinity (Sazonov et al. 2006).

It is important to emphasize that the spectral shape of the emission of faint discrete sources is very similar to the emission of multitemperature hot interstellar gas and it is virtually impossible to distinguish one from the other using present-day $\mathrm{X}$-ray spectrometers. We may anticipate, however, that the next generation of high energy resolution X-ray spectrometers will be able to disntinguish these two types of sources by detecting the expected redshifted lines in the emission from the accreting white dwarf in CVs or through the expected differences in the line emission of the relatively dense plasma in stellar coronae and in the tenuous interstellar gas.

We showed that the relative contributions of different constituents to the total emission of galaxies are expected to strongly depend on the energy band of the given study. We presented schematic broad-band spectra of the major contributors to the $\mathrm{X}$-ray emission of galaxies (see Fig. 7).

The fact that the cumulative contribution of weak stellar-type $\mathrm{X}$-ray sources can provide a significant or even dominant contribution to the observed unresolved extended X-ray emission of galaxies even as massive as NGC $3379\left(\sim 6 \times 10^{10} M_{\odot}\right)$ must be considered in different studies such as measurements of the masses and the hot gas content of elliptical galaxies.

Acknowledgements. This research made use of data obtained from the High Energy Astrophysics Science Archive Research Center Online Service, provided by the NASA/Goddard Space Flight Center. This publication makes use of data products from the Two Micron All Sky Survey, which is a joint project of the University of Massachusetts and the Infrared Processing and Analysis Center/California Institute of Technology, funded by the National Aeronautics and Space Administration and the National Science Foundation. This work was supported by DFG-Schwerpunktprogramme (SPP 1177), grants CH389/3-2, RFFI 07-02-01051, 07-02-00961, NSH-5579.2008.2 and by program of Presidium of Russian Academy of Sciences "Formation and evolution of stars and galaxies".

\section{References}

Anders, E., \& Grevesse, N. 1989, GeCoA, 53, 197

Bell, E. F., McIntosh, D. H., Katz, N., \& Weinberg, M. D. 2003, ApJS, 149, 289

Bîrzan, L., Rafferty, D. A., McNamara, B. R., Wise, M. W., \& Nulsen, P. E. J. 2004, ApJ, 607, 800

Bogdan, A., \& Gilfanov, M. 2008 [arXiv:0803.0063]

Bohringer, H., Briel, U. G., Schwarz, R. A., et al. 1994, Nature, 368, 828

Churazov, E., Brüggen, M., Kaiser, C. R., Böhringer, H., \& Forman, W. 2001, ApJ, 554, 261

David, L. P., Jones, C., Forman, W., \& Murray, S. S. 2005, ApJ, 635, 1053

Dickey, J. M., \& Lockman, F. J. 1990, ARA\&A, 28, 215

Eker, Z., Demircan, O., Bilir, S., \& Karataş, Y. 2006, MNRAS, 373, 1483

Forman, W., Jones, C., \& Tucker, W. 1985, ApJ, 293, 102

Fukazawa, Y., Botoya-Nonesa, J. G., Pu, J., Ohto, A., \& Kawano, N. 2006, ApJ, 636, 698

Gilfanov, M. 2004, MNRAS, 349, 146

Heckman, T. M., Armus, L., \& Miley, G. K. 1990, ApJS, 74, 833

Irwin, J. A., \& Sarazin, C. L. 1998, ApJ, 499, 650

Irwin, J. A., Athey, A. E., \& Bregman, J. N. 2003, ApJ, 587, 356

Jarrett, T. H., Chester, T., Cutri, R., Schneider, S. E., \& Huchra, J. P. 2003, AJ, 125,525

Jensen, J. B., Tonry, J. L., Barris, B. J., et al. 2003, ApJ, 583, 712

Krivonos, R., Revnivtsev, M., Churazov, E., et al. 2007, A\&A, 463, 957

Mitsuda, K., Inoue, H., Koyama, K., et al. 1984, PASJ, 36, 741

Li, Z., \& Wang, Q. D. 2007, ApJ, 668, L39

Loewenstein, M., \& Mathews, W. G. 1991, ApJ, 373, 445

Paczynski, B., \& Sienkiewicz, R. 1981, ApJ, 248, L27

Pavlinsky, M. N., Grebenev, S. A., \& Sunyaev, R. A. 1994, ApJ, 425, 110

Pellegrini, S., \& Fabbiano, G. 1994, ApJ, 429, 105

Pellegrini, S., \& Ciotti, L. 2006, MNRAS, 370, 1797

Pellegrini, S., Baldi, A., Kim, D. W., et al. 2007, ApJ, 667, 731

Rappaport, S., Joss, P. C., \& Webbink, R. F. 1982, ApJ, 254, 616

Revnivtsev, M., Sazonov, S., Gilfanov, M., Churazov, E., \& Sunyaev, R. 2006, A\&A, 452, 169

Revnivtsev, M., Churazov, E., Sazonov, S., Forman, W., \& Jones, C. 2007, A\&A, 473,783

Sazonov, S., Revnivtsev, M., Gilfanov, M., Churazov, E., \& Sunyaev, R. 2006, A\&A, 450, 117

Shirey, R., Soria, R., Borozdin, K., et al. 2001, A\&A, 365, L195

Strickland, D. K., Heckman, T. M., Weaver, K. A., \& Dahlem, M. 2000, AJ, 120, 2965

Strickland, D. K., Heckman, T. M., Colbert, E. J. M., Hoopes, C. G., \& Weaver, K. A. 2004, ApJS, 151, 193

Trinchieri, G., \& Fabbiano, G. 1985, ApJ, 296, 447

Vikhlinin, A., McNamara, B. R., Forman, W., et al. 1998, ApJ, 502, 558

Vikhlinin, A., Markevitch, M., Murray, S. S., et al. 2005, ApJ, 628, 655

Walter, F. M., \& Bowyer, S. 1981, ApJ, 245, 671 